Pacific Journal of Mathematic 


\title{
NOTES ON COMMUTATIVE POWER JOINED SEMIGROUPS
}

\author{
Richard G. Levin and TAKayuki TAMURA
}

Let $S$ be a commutative semigroup. The main theorem in this paper is to prove that the following two conditions are equivalent: (1) For all $a, b \in S$ there are positive integers $m$, $n$ such that $a^{m}=b^{n}$. (2) For all $a, b \in S, a^{l}=a^{m} b^{n}, b^{r}=$ $b^{s} a^{t}$ for some $l, m, n, r, s, t$. As a consequence of the theorem, the authors prove that a commutative archimedean semigroup $S$ without idempotent is power joined if and only if the structure group of $S$ is a torsion group.

Let $S$ be a commutative archimedean semigroup without idempotent. Consider the following question: "Under what condition on the structure group (defined below) of $S$ will $S$ be power joined?" Levin proved in [4] that if $S$ is finitely generated, equivalently if the structure group of $S$ is finite, then $S$ is power joined. Also he obtained a necessary and sufficient condition for $S$ to be power joined. The following is Theorem 2 in [4]:

THEOREM 1. Let $S$ be a commutative, archimedean semigroup without idempotent. Let $G_{a}=S / \rho_{a}$ be the structure group of $S$ determined by a. Then $S$ is power joined if and only if $G_{a}$ is periodic and the congruence class containing a modulo $\rho_{a}$ is power joined.

If we assume that $S$ is additionally cancellative, that is, $S$ is an $\mathfrak{R}$-semigroup, then the answer is simple. The following is due to Chrislock $[1,2]$.

Theorem 2. An $\mathfrak{R}$-semigroup $S$ is power joined if and only if $G_{a}$ is periodic for some $a \in S$, equivalently for all $a \in S$.

Naturally the following question is raised: Can Theorem 1 be improved such that Theorem 2 is extended to $S$ in Theorem 1? The question is affirmative. In this paper we study the problem for more general case, i.e., for commutative archimedean semigroups. The main theorem of this paper asserts that a commutative semigroup $S$ is power joined if and only if it is archimedean and its group homomorphic images are periodic. As a corollary we can answer the above question.

Semigroups are assumed to be commutative throughout this paper. 
Definition 1. A semigroup $S$ is called power joined if and only if for all $a, b \in S$, there are positive integers $n, m$ such that

$$
a^{n}=b^{m} \text {. }
$$

Definition 2. A semigroup $S$ is called archimedean if and only if for all $a, b \in S$, there exist $u, v \in S$ and positive integers $n, m$ such that

$$
a^{n}=b u \quad \text { and } \quad b^{m}=a v .
$$

DEFINITION 3. Let $S$ be an archimedean semigroup without idempotent. We define a congruence $\rho_{b}$ on $S$ for fixed $b \in S$ as follows. We define $x \rho_{b} y$ if and only if there are positive integers $n$ and $m$ such that

$$
b^{n} x=b^{m} y
$$

REMARK. More information on commutative, archimedean semigroups without idempotent can be found in [1], [6] and [7]. In particular a proof that $\rho_{b}$ (as defined above) is a congruence relation and that $S / \rho_{b}=G_{b}$ is a group can be found in [7]. $S / \rho_{b}$ is called the structure group of $S$ determined by $b$. Also notice $x y \neq y$ for all $x, y \in S$.

THEOREM 3. The following statements are equivalent.

(3.1) The semigroup $S$ is power joined.

(3.2) The semigroup $S$ is archimedean and its group homomorphic images are periodic.

(3.3) The semigroup $S$ satisfies the conditions: for all pairs $a, b \in S$, there are positive integers $l, m, n, s, t, p$ such that

$$
a^{l}=a^{m} b^{n} \quad \text { and } \quad b^{s}=b^{t} a^{p} \text {. }
$$

Proof. We will prove: $(3.1) \Rightarrow(3.2) \Rightarrow(3.3) \Rightarrow(3.1)$. Let $S$ be a power joined semigroup. It is trivial to show that $S$ is archimedean. Let $G$ be a group homomorphic image of $S$ with $\varphi: S \rightarrow G$ the homomorphism. We will show that $G$ is a periodic group. Let $a \in G$ and let $e$ be the identity of $G$. There exist $x, y \in S$ such that $\varphi(x)=a, \varphi(y)=e$. Since $S$ is power joined, there exist positive integers $n, m$ such that $x^{n}=y^{m}$. Then

$$
a^{n}=[\varphi(x)]^{n}=\varphi\left(x^{n}\right)=\varphi\left(y^{m}\right)=[\varphi(y)]^{m}=e^{m}=e .
$$

We see that $G$ is periodic and this completes the proof that $(3.1) \Rightarrow$ (3.2). 
We next prove that $(3.2) \Rightarrow(3.3)$. Let $S$ be an archimedean semigroup whose group homomorphic images are periodic.

Case 1. Assume that $S$ has an idempotent $e$. Then the set $S e$ is a group and is the homomorphic image of $S$ (see [3] or [5]). Let $a, b \in S$. Then $a e$ and $b e$ are elements of $S e$. Since $S e$ is a periodic group with $e$ as its identity element, there exist positive integers $n$ and $m$ such that

$$
(a e)^{n}=e \quad \text { and } \quad(b e)^{m}=e .
$$

That is,

$$
a^{n} e=e=b^{m} e .
$$

Since $S$ is archimedean, there exist positive integers $k$ and $t$ and $u, v \in S$ such that

$$
a^{k}=e v \quad \text { and } \quad b^{t}=e u .
$$

From equations (1) and (2) we derive

$$
\begin{aligned}
a^{n} e u & =b^{m} e u, \\
\text { or } a^{n} b^{t} & =b^{m} b^{t}, \\
\text { or } a^{n} b^{t} & =b^{r} \quad \text { where } \quad \mathrm{r}=m+t .
\end{aligned}
$$

Similarly, we derive $a^{l}=a^{k} b^{m}$ for some positive integers $l, k$ and $m$.

Case 2. Assume that $S$ does not have an idempotent. Let $a, b \in S$. Consider the congruence $\rho_{a}$ of Definition 3. Then $S / \rho_{a}$ is a group homomorphic image of $S$ and, therefore, is a periodic group. Also

$$
S=\bigcup_{\lambda \in S / \rho_{a}} S_{\lambda}
$$

and $a \in S_{\varepsilon}$, where $\varepsilon$ is the identity of $S / \rho_{a}$. There is $\lambda \in S / \rho_{a}$ such that $b \in S_{\lambda}$. There exists a positive integer $k$ such that $\lambda^{k}=\varepsilon$. Thus,

$$
b^{k} \in S_{\lambda^{k}}=S_{\varepsilon} .
$$

That is, $a$ and $b^{k}$ are $\rho_{a}$ related. By definition of $\rho_{a}$, there are positive integers $n$ and $m$ such that

$$
\begin{aligned}
a^{n} a & =a^{m} b^{k}, \\
\text { or } a^{l} & =a^{m} b^{k}, \quad \text { where } l=n+1 .
\end{aligned}
$$

Similarly, we can derive the equation

$$
b^{s}=b^{t} a^{p} .
$$


The proof that $(3.2) \Rightarrow(3.3)$ is now complete.

We now prove that $(3.3) \Rightarrow(3.1)$.

Case 1. Assume that $S$ has an idempotent $e$. Let $a \in S$. Then there are positive integers $l, m, n, s, t$ and $p$ such that

$$
\begin{aligned}
e^{l} & =e^{m} a^{n} \quad \text { and } \quad a^{s}=a^{t} e^{p}, \\
\text { or } e & =e a^{n} \quad \text { and } \quad a^{s}=a^{t} e .
\end{aligned}
$$

Using the equations of (4) we derive

$$
e=e^{t}=\left(e a^{n}\right)^{t}=e^{t}\left(a^{t}\right)^{n}=\left(e a^{t}\right)^{n}=\left(a^{s}\right)^{n} .
$$

Thus, we have $e=a^{r}$ for a positive integer $r$.

It is now obvious that if $a, b \in S$, there are positive integers $u$ and $v$ such that $a^{u}=b^{v}$. Therefore $S$ is power joined.

Case 2. Assume that $S$ has no idempotent. Again we have for any pair $a, b \in S$, positive integers $l, m, n, s, t$ and $p$ such that

$$
a^{l}=a^{m} b^{n} \quad \text { and } \quad b^{s}=b^{t} a^{p} .
$$

We will prove that there are positive integers $l^{\prime}$ and $n^{\prime}$ such that $a^{l^{\prime}}=a^{m} b^{n^{\prime}}$, and $n^{\prime} p \geqq m t$. Since $S$ does not have an idempotent, $l>m$ in (5). Then

$$
a^{2 l-m}=a^{l-m} a^{l}=a^{l-m} a^{m} b^{n}=a^{l} b^{n}=\left(a^{m} b^{n}\right) b^{n}=a^{m} b^{2 n} .
$$

Now assume that for some integer $k \geqq 1$, we have

$$
a^{k l-(k-1) m}=a^{m} b^{k n} .
$$

We will prove that

$$
a^{(k+1) l-k m}=a^{m} b^{(k+1) n} .
$$

Now we have

$$
\begin{aligned}
a^{(k+1) l-k m} & =a^{k l-k m} a^{l}=a^{k l-k m}\left(a^{m} b^{n}\right)=\left(a^{k l-k m} a^{m}\right) b^{n} \\
& =a^{k l-(k-1) m} b^{n}=\left(a^{m} b^{k n}\right) b^{n}=a^{m} b^{(k+1) n} .
\end{aligned}
$$

Thus, by induction we have the relation: for every $k \geqq 1$

$$
a^{k l-(k-1) m}=a^{m} b^{k n} .
$$

Now choose $k$ such that $k n p \geqq m t$. Set $n^{\prime}=k n, l^{\prime}=k l-(k-1) m$. We replace the equations of (5) by the equations

$$
a^{l^{\prime}}=a^{m} b^{n^{\prime}} \text { and } b^{s}=b^{t} a^{p} .
$$


From (6) we derive

$$
\begin{aligned}
a^{l^{\prime} t p} & =\left(b^{n^{\prime}}\right)^{t p}\left(a^{m}\right)^{t p}=\left(b^{t}\right)^{n^{\prime} p}\left(a^{p}\right)^{m t}, \\
\text { or } a^{l^{\prime} t p} & =\left(b^{t}\right)^{m t+\left(n^{\prime} p-m t\right)}\left(a^{p}\right)^{m t} \\
& =\left(b^{t}\right)^{m t}\left(b^{t}\right)^{n^{\prime} p-m t}\left(a^{p}\right)^{m t} \\
& =\left(b^{t} a^{p}\right)^{m t}\left(b^{t}\right)^{n^{\prime} p-m t} \\
& =\left(b^{s}\right)^{m t}\left(b^{t}\right)^{n^{\prime} p-m t} .
\end{aligned}
$$

Set $u=l^{\prime} t p$ and $v=s m t+t\left(n^{\prime} p-m t\right)$. We see that we have derived the equation $a^{n}=b^{v}$. Therefore $S$ is power joined. This concludes the proof that $(3.3) \Rightarrow(3.1)$.

REMARK. Each of (3.1), (3.2) and (3.3) is equivalent to one of (3.4) and (3.5) below:

(3.4) The semigroup $S$ satisfies the following condition: there is an element $a_{0}$ of $S$ such that for all $b \in S$ there are positive integers $l, m, n, s, t, p$ satisfying

$$
a_{0}^{l}=a_{0}^{m} b^{n} \text { and } b^{s}=b^{t} a_{0}^{p} .
$$

(3.5) The semigroup $S$ satisfies the condition: for all pairs $a, b \in S$ there are positive integers $l, m, s, t$ such that

$$
a^{l}=(a b)^{m} \text { and } b^{s}=(b a)^{t} .
$$

Proof. We define a relation $\tau$ on $S$ as follows:

$a \tau b$ if and only if $a^{l}=a^{m} b^{n}$ and $b^{s}=b^{t} a^{p}$ for some $l, m, n, s, t, p$. Then $\tau$ is an equivalence on $S$. Reflexivity and symmetry are obvious. Transitivity is proved as follows: suppose $a^{l}=a^{m} b^{n}$ and $b^{k}=b^{q} c^{v}$. First we have

$$
a^{l k}=a^{m k} b^{n k}=a^{m k} b^{n q} c^{n v}
$$

and then

$$
a^{l^{\prime}}=a^{m^{\prime}}\left(a^{m q} b^{n q}\right) c^{n v}=a^{m^{\prime}+l q} c^{n v}
$$

where

$$
\begin{aligned}
& l^{\prime}=l k, m^{\prime}=m k-m q \quad \text { if } \quad k \geqq q \\
& l^{\prime}=l k=m q-m k, m^{\prime}=0 \text { if } k<q .
\end{aligned}
$$

Therefore $(3.4) \Rightarrow(3.3)$ is obtained as an immediate consequence; $(3.3) \rightarrow(3.4),(3.1) \rightarrow(3.5)$ and $(3.5) \rightarrow(3.3)$ are obvious.

If $S$ is a nil-semigroup, i.e., a semigroup in which some power of every element is zero, Theorem 3 is trivial since every nil-semigroup is power joined.

If $S$ is an archimedean semigroup whose idempotent is not zero, 
then $G=S e$ is the kernel, i.e., the minimal ideal and the unique maximal subgroup. Then we have

COROLLARY 5. $S$ is power joined if and only if the kernel $G$ is periodic.

The essense of Theorem 3 is in the case where $S$ is an archimedean semigroup without idempotent.

THEOREM 6. An archimedean semigroup without idempotent is power joined if and only if the structure group $G_{a}=S / \rho_{a}$ of $S$ is periodic for some $a \in S$, equivalently for all $a \in S$.

Proof. Let $S$ be an archimedean semigroup without idempotent. Then the statement (3.3) is equivalent to:

$$
S / \rho_{a} \text { is periodic for all } a \in S \text {. }
$$

(3.4) is equivalent to:

$$
S / \rho_{a} \text { is periodic for some } a \in S .
$$

The first statement is obvious. To see the second we will prove the following:

If $S / \rho_{a_{0}}$ is periodic, then for all $b \in S$ there are positive integers $l, m, n, s, t, p$ such that

$$
a_{0}^{l}=a_{0}^{m} b^{n}, \quad b^{s}=b^{t} a_{0}^{p} .
$$

The first of (7) is immediately obtained. Since $S$ is archimedean there is a positive integer $k$ and an element $c \in S$ such that

$$
b^{k}=a_{0} c
$$

which implies $b^{k l}=a_{0}^{l} c^{l}$. Since $S$ has no idempotent, $l>m$ in the first of (7). Now we have

$$
b^{k l} a_{0}^{l-m}=a_{0}^{l-m} a_{0}^{l} c^{l}=a_{0}^{l-m} a_{0}^{m} b^{n} c^{l}=b^{n} a_{0}^{l} c^{l}=b^{n} b^{k l}=b^{n+k l} .
$$

This completes the proof.

\section{BIBLIOGRAPHY}

1. James Chrislock, The structure of archimedean semigroups, Thesis, University of California, Davis, 1966.

2. — On medial semigroups, J. of Algebra 12 (1969), 1-9.

3. A. H. Clifford and G. B. Preston, The algebraic theory of semigroups, vol. 1, Math. Surveys No. 7, Amer. Math. Soc., Providence, R. I., 1961.

4. Richard Levin, On commutative, nonpotent, archimedean semigroups, Pacific J. 
Math. 27 (1968), 365-371.

5. Takayuki Tamura, Note on unipotent inversible semigroups, Kodai Math. Sem. Reports (1954), 93-95.

6. - Commutative nonpotent archimedean semigroups with cancellation law 1 , J. of Gakugei, Tokushima Univ. 8 (1951), 5-11.

7. - Construction of trees and commutative archimedean semigroups, Math. Nachr. 36 (1968), 255-287.

Received October 9, 1969, and in revised form June 9, 1970. The research for this paper was supported in part by NSF, GP-11964; a part of the results of this paper was presented by $R$. Levin in the meeting of the American Mathematical Society which was held in Santa Cruz, California, on April 26, 1969.

Western Washington State College, Bellingham, Washington AND

University of California, Davis, California 



\section{PACIFIC JOURNAL OF MATHEMATICS}

\section{EDITORS}

\author{
H. SAMELSON \\ Stanford University \\ Stanford, California 94305 \\ Richard Pierce \\ University of Washington \\ Seattle, Washington 98105
}

\author{
J. DugundJI \\ Department of Mathematics \\ University of Southern California \\ Los Angeles, California 90007 \\ RICHARD ARENS \\ University of California \\ Los Angeles, California 9.0024
}

\section{ASSOCIATE EDITORS}

\begin{tabular}{|c|c|}
\hline E. F. BECKENBACH & K. YoshidA \\
\hline \multicolumn{2}{|c|}{ SUPPORTING INSTITUTIONS } \\
\hline UNIVERSITY OF BRITISH COLUMBIA & STANFORD UNIVERSITY \\
\hline CALIFORNIA INSTITUTE OF TECHNOLOGY & UNIVERSITY OF TOKYO \\
\hline UNIVERSITY OF CALIFORNIA & UNIVERSITY OF UTAH \\
\hline MONTANA STATE UNIVERSITY & WASHINGTON STATE UNIVERSITY \\
\hline UNIVERSITY OF NEVADA & UNIVERSITY OF WASHINGTON \\
\hline NEW MEXICO STATE UNIVERSITY & $* \quad * \quad *$ \\
\hline OREGON STATE UNIVERSITY & AMERICAN MATHEMATICAL SOCIETY \\
\hline UNIVERSITY OF OREGON & CHEVRON RESEARCH CORPORATION \\
\hline OSAKA UNIVERSITY & TRW SYSTEMS \\
\hline UNIVERSITY OF SOUTHERN CALIFORNIA & NAVAL WEAPONS CENTER \\
\hline
\end{tabular}

The Supporting Institutions listed above contribute to the cost of publication of this Journal, but they are not owners or publishers and have no responsibility for its content or policies.

Mathematical papers intended for publication in the Pacific Journal of Mathematics should be in typed form or offset-reproduced, (not dittoed), double spaced with large margins. Underline Greek letters in red, German in green, and script in blue. The first paragraph or two must be capable of being used separately as a synopsis of the entire paper. The editorial "we" must not be used in the synopsis, and items of the bibliography should not be cited there unless absolutely necessary, in which case they must be identified by author and Journal, rather than by item number. Manuscripts, in duplicate if possible, may be sent to any one of the four editors. Please classify according to the scheme of Math. Rev. Index to Vol. 39. All other communications to the editors should be addressed to the managing editor, Richard Arens, University of California, Los Angeles, California, 90024.

50 reprints are provided free for each article; additional copies may be obtained at cost in multiples of 50 .

The Pacific Journal of Mathematics is published monthly. Effective with Volume 16 the price per volume (3 numbers) is $\$ 8.00$; single issues, $\$ 3.00$. Special price for current issues to individual faculty members of supporting institutions and to individual members of the American Mathematical Society: $\$ 4.00$ per volume; single issues $\$ 1.50$. Back numbers are available.

Subscriptions, orders for back numbers, and changes of address should be sent to Pacific Journal of Mathematics, 103 Highland Boulevard, Berkeley, California, 94708.

PUBLISHED BY PACIFIC JOURNAL OF MATHEMATICS, A NON-PROFIT CORPORATION

Printed at Kokusai Bunken Insatsusha (Internatıonal Academic Printing Co., Ltd.), 7-17, Fujimi 2-chome, Chiyoda-ku, Tokyo, Japan. 


\section{Pacific Journal of Mathematics}

\section{Vol. 35, No. $3 \quad$ November, 1970}

John D. Arrison and Michael Rich, On nearly commutative degree one algebras . . . 533

Bruce Alan Barnes, Algebras with minimal left ideals which are Hilbert spaces . . . . 537

Robert F. Brown, An elementary proof of the uniqueness of the fixed point index . . . 549

Ronn L. Carpenter, Principal ideals in F-algebras .................... 559

Chen Chung Chang and Yiannis (John) Nicolas Moschovakis, The Suslin-Kleene

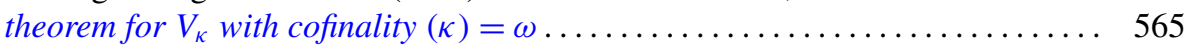

Theodore Seio Chihara, The derived set of the spectrum of a distribution

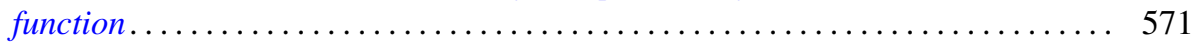

Tae Geun Cho, On the Choquet boundary for a nonclosed subspace of $C(S) \ldots \ldots \quad 575$

Richard Brian Darst, The Lebesgue decomposition, Radon-Nikodym derivative,

conditional expectation, and martingale convergence for lattices of sets .......

David E. Fields, Dimension theory in power series rings . . . . . . . . . . . .

Michael Lawrence Fredman, Congruence formulas obtained by counting

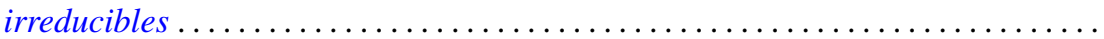

John Eric Gilbert, On the ideal structure of some algebras of analytic functions.....

G. Goss and Giovanni Viglino, Some topological properties weaker than

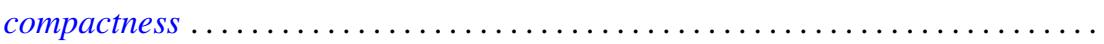

581

601

625

George Grätzer and J. Sichler, On the endomorphism semigroup (and category) of

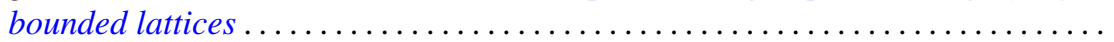

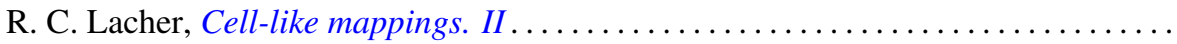

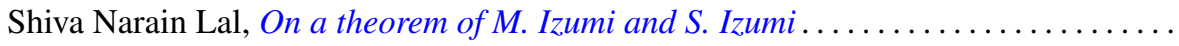

661

Howard Barrow Lambert, Differential mappings on a vector space ...............

Richard G. Levin and Takayuki Tamura, Notes on commutative power joined

semigroups.

Robert Edward Lewand and Kevin Mor McCrimmon, Macdonald's theorem for quadratic Jordan algebras.

J. A. Marti, On some types of completeness in topological vector spaces ....

Walter J. Meyer, Characterization of the Steiner point

717

Saad H. Mohamed, Rings whose homomorphic images are $q$-rings ...

727

Thomas V. O'Brien and William Lawrence Reddy, Each compact orientable surface

of positive genus admits an expansive homeomorphism ...

737

Robert James Plemmons and M. T. West, On the semigroup of binary relations...

743

Calvin R. Putnam, Unbounded inverses of hyponormal operator . .

755

William T. Reid, Some remarks on special disconjugacy criteria for differential

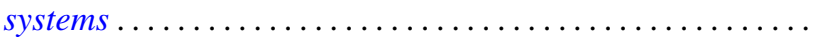

C. Ambrose Rogers, The convex generation of convex Borel sets in euclidean

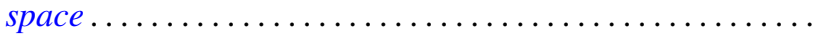

S. Saran, A general theorem for bilinear generating functions .

S. W. Smith, Cone relationships of biorthogonal systems ......

Wolmer Vasconcelos, On commutative endomorphism rings ....

795

Vernon Emil Zander, Products of finitely additive set functions from Orlicz

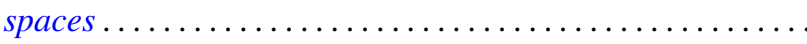

G. Sankaranarayanan and C. Suyambulingom, Correction to: "Some renewal

theorems concerning a sequence of correlated random variables" .

Joseph Zaks, Correction to: "Trivially extending decompositions of $E^{n}$ ”....... 805

Dong Hoon Lee, Correction to: "The adjoint group of Lie groups" ............ 805

James Edward Ward, Correction to: "Two-groups and Jordan algebras". 